\title{
HYPOLIPIDEMIC ACTIVITY OF AMINE-BORANE ADUCTS OF CYCLOHEXYLAMINE AND TOLUIDINE IN RODENTS
}

\author{
Bruce S. Burnham', S.Y. Chen', A. Sood², Bernard F. Spielvogel2 and Iris H. Hall' \\ I Division of Medicinal Chemistry and Natural Products, School of Pharmacy, \\ University of North Carolina, Chapel Hill, N.C, 27599-7360, USA \\ 2 Boron Biologicals, Inc., 620 Hutton Rd., Raleigh, N.C. 27606, USA
}

\begin{abstract}
The amine-borane adducts of cyclohexylamine and toluidine were observed to be potent hypolipidemic agents in mice, I.P. and rats orally at 8 $\mathrm{mg} / \mathrm{kg} /$ day lowering both serum cholesterol and triglyceride levels after 14-16 days. These compounds were able to lower tissue lipids including the cholesterol content of the aorta wall. The agents successfully lower VLDL- and LDL-cholesterol content while elevating HDL-cholesterol content significantly. The agents also modulate lipid regulatory enzyme activities in a manner to reduce liver lipid levels. These studies demonstrate that the nitrogen atom does not have to be apart of the aromatic ring as in heterocyclic-amine borane to afford good hypolipidemic activity in rodents.
\end{abstract}

\section{INTRODUCTION}

Previously we have examined a number of amine-cyanoboranes[1], aminecarboxyboranes and their amides and esters[2-5], di- and tri-peptide boranes [6], heterocyclic-amine boranes [7], polyborate salts [8], choline and thiocholine boranes[9], phosphoacetate boranes[10] and

deoxyribonucleoside cyanoboranes[11]. These agents were effective in rodents at $8 \mathrm{mg} / \mathrm{kg} /$ day, significantly lowering both serum cholesterol and triglyceride levels after 14-16 days. Cholesterol levels were lowered in rat VLDL and LDL fractions while the HDL cholesterol levels were markedly elevated. The mode of action of these derivatives was to acclerate bile cholesterol excretion and alteration of regulatory enzyme activities involved in de novo lipid synthesis. Furthermore, sub-acute toxicity studies in mice indicated no organ specific toxicity and mean survival doses were high[12]. Thus, we have decided to examine a series cyano- and carboxyborane adducts of cyclohexylamine and toluidine for their hypolipidemic activity in rodents. These studies should determine if the nitrogen atom is necessary in the aromatic ring to maintain activity as a hypolipidemic agent in rodents.

\section{MATERIALS and METHODS}

Source of compounds

All of the compounds were synthesized previously and chemically and physically charecterized[13]: cyclohexylamine-cyanoborane $\underline{1}$, cyclohexylamine-carboxyborane $\underline{2}, \mathrm{~N}$-methylcyclohexylamine-carboxyborane $\underline{3}, \mathrm{~N}$-ethylcyclohexylamine-carboxyborane $\underline{4}, \mathrm{~N}, \mathrm{~N}$-dimethylcyclohexylaminecarboxyboranes $\underline{5}, 2$-methylcyclohexylamine-carboxyborane $\underline{6}, 3$ methylcyclohexylamine-carboxyborane $\mathbf{7}, 4$-methylcyclohexylaminecarboxyborane $\underline{8}, \mathrm{~N}$-ethylcyclohexylamine-cyanoborane $\underline{9}, \mathrm{~N}$ - 
methylcyclohexylamine-cyanoborane 10, N,N-dimethylcyclohexylaminecyanoborane 11, 2-methylcyclohexylamine-cyanoborane 12, 3methylcyclohexylamira-cyanoborane 13 , 4- methylcyclohexylamminecyanoborane 14, 2,3-dimethylcyclohexylamine-cyanoboranes 15, ㅇtoluidine-cyanoborane 16 , m-toluidine-cyanoborane 17, p-toluidinecyanoborane 18 , and p-anisidine cyanoborane 19. All isotopes were purchased from New England Nuclear. Substrates and co-factors were obtained from Sigma Chemical Co. Sprague Dawley male rats were obtained from Charles River Laboratory. $\mathrm{CF}_{1}$ mice were obtained from Jackson Laboratory. Animals were maintained in light cycles of $12 \mathrm{~h}$ at $22^{\circ} \mathrm{C}$. Rats were maintained in individual wire cages and mice were housed three/plastic cage. Food (Agway/Prolab Animal Diet) and water were ad libitum.

Normolipidemic studies

For structure activity studies, $\mathrm{CF}_{1}$ male mice $(\sim 28 \mathrm{~g})$ were administered amine-borane adducts of cyclohexylamine and toluidine in 18 CMC at 8 $\mathrm{mg} / \mathrm{kg} /$ day, I.P. Blood samples were obtained on days 9 and 16 between 7:30 and 8:30 a.m. Daily dosing of the agents was between 9:00 and 10:00 a.m. the serum was obtained by centrifuging the blood for $10 \mathrm{~min}$. at $3500 \mathrm{~g}$. The serum cholesterol levels were determined by a modification of the procedure Liebermann-Burchard reaction [14]. Serum triglyceride were determined using a commercial kit [Boehringer Mannheim Diagnostics]. Sprague Dawley male rats $(-230 \mathrm{~g})$ were administered orally Compounds $\underline{7}, \underline{9}$ or $\underline{18}$ at $8 \mathrm{mg} / \mathrm{kg} /$ day, for two weeks. Weekly blood samples were obtained by tail vein bleeding.

Animal weight, organ weight and food consumption Control and treated normolipidemic Sprague Dawley male rat $(\sim 230 \mathrm{~g})$ weights were obtained and expressed as a percentage of the initial body weight (week zero). Food consumption (gm/day/rat) was noted for two weeks for control and treated rats[15].

Tissue lipid levels Normolipidemic Sprague Dawley male rats $(\sim 230 \mathrm{~g})$ which were treated orally for two weeks with compound $\underline{7}, \underline{9}$ or 18 at $8 \mathrm{mg} / \mathrm{kg} /$ day, were sacrificed and tissue samples of the liver, small intestinal mucosa and aorta were removed. A $24 \mathrm{hr}$ fecal sample was also obtained. A $10 \%$ homogenate in $0.25 \mathrm{M}$ sucrose $+0.001 \mathrm{M}$ EDTA, $\mathrm{pH} 7.2$, was prepared for each tissue. An aliquot (2 $\mathrm{ml}$ ) of the homogenate was extracted[16-17] and the number of $\mathrm{mg}$ of lipid extracted was weighed. The lipid residue was taken up in methylene chloride and the levels of cholesterol [14], triglycerides, neutral lipids[18] and phospholipids[19] were determined. Protein content of the whole homogenate was determined[20].

Serum lipoprotein fractions

Normolipidemic Sprague Dawley male rats $(230 \mathrm{~g})$ treated for two weeks with compounds $\underline{7}, \underline{9}$ or $\underline{18}$ at $8 \mathrm{mg} / \mathrm{kg} /$ day, orally were anesthesized with ether and blood $(-10 \mathrm{ml})$ was collected from the abdominal vein. Serum was separated from whole blood by centrifugation at $3500 \mathrm{rpm}$. Aliquots of the serum were separated into chylomicrons, VLDL, HDL and LDL by ultracentrifugation as modified for normal rats[21-22]. Each of the fractions was analyzed for cholesterol, triglyceride, neutral lipids, phospholipid and protein levels. 
Enzymatic studies

In vitro enzymatic studies were performed using $10 \%$ homogenates of liver mucosa from normolipidemic Sprague Dawley male rats $(\sim 280 \mathrm{~g})$. The liver homogenates were prepared in $0.25 \mathrm{M}$ sucrose $+0.001 \mathrm{M}$ (ethylenedinitrilo)tetraacetic acid [EDTA], $\mathrm{pH}$ 7.2. Acetyl coenzyme A synthetase[23] and adenosine triphosphate dependent citrate lyase activities[24] were determined spectrophotometrically at $540 \mathrm{~nm}$ as the hydroxylamate of acetyl coenzyme A formed after $20 \mathrm{~min}$ at $37^{\circ} \mathrm{C}$. Cholesterol- $7 \alpha$-hydroxylase activity was determined using [1,2${ }^{3} \mathrm{H}$ ]cholesterol ( $60 \mathrm{mCi} / \mathrm{mmol}$ ) [25], and acyl CoA cholesterol acyl transferase activity was determined using $[1-14 \mathrm{C}]$ oleic acid (56.7 $\mathrm{mCi} / \mathrm{mmol}$ ) [26]. Cholesterol synthesis was measured using [ $1-{ }^{14} \mathrm{C}$ ] acetyl CoA (62 $\mathrm{mCi} / \mathrm{mmol}$ ) ard a post-mitochondrial supernatant (9000 g x $20 \mathrm{~min}$ ) which was incubated for $60 \mathrm{~min}$ at $37^{\circ} \mathrm{C} \mathrm{[27].} \mathrm{The} \mathrm{digitonide} \mathrm{derivative}$ of cholesterol was isolated and counted [28]. Cholesterol ester hydrolase activity was determined using $1-{ }^{14} \mathrm{C}$ cholesterol oleate [56.6 $\mathrm{mCi} / \mathrm{mol}$ ] [29].

For acetyl coenzyme A carboxylase activity, the enzyme had to be polymerized for $30 \mathrm{~min}$ at $37^{\circ} \mathrm{C}$ and then the assay mixture containing sodium ${ }^{14} \mathrm{C}$-bicarbonate $(41.0 \mathrm{mCi} / \mathrm{mmol})$ was added and incubated for 30 min at $37^{\circ} \mathrm{C}$ with test drugs[30]. sn-Glycerol-3-phosphate acyl transferase activity was determined with sn-glycerol-3-phosphate [L-2$3 \mathrm{H}(\mathrm{N})]$ ( $7.1 \mathrm{Ci} / \mathrm{mmol}$ ) with the microsomal fraction of liver homogenates. The reaction was terminated after $60 \mathrm{~min}$ and the lipids were extracted with chloroform/methanol (2:1) containing $1 \% \mathrm{HCl}$ and counted[31]. Phosphatidylate phosphohydrolase activity was measured as inorganic phosphate released after $60 \mathrm{~min}[32]$. The released inorganic phosphate after color development with ascorbic acid and ammonium molybate was quantitated at $820 \mathrm{~nm}$. Hepatic lipoprotein lipase was determined using glycerol-tri-14 C-palmitate [64 mCi/mol] emulsified with lecithin by the method of Chait et a1.[33]. Protein content of the liver homogenates was determined[20].

Data displayed in Tables $1-5$ represent means \pm standard deviations. The Student's "t" test was applied between control groups and the individual drug treatment groups using the raw data. The analysis of variance (ANOVA) was applied among test drugs and is reported in the text only.

\section{RESULTS}

Selected carboxy- and cyanoborane adducts of cyclohexylamine and toluidine were shown to be significantly active in the structure activity study in mice at $8 \mathrm{mg} / \mathrm{kg} /$ day. In comparison to the standards lovastatin and clofibrate, compounds $\underline{1}, \underline{2}, \underline{5}, \underline{7}, \underline{8}, \underline{9}, \underline{10}, \underline{13}, \underline{16}, \underline{17}$, and 18 lowered serum cholesterol by $23 \%$ at $8 \mathrm{mg} / \mathrm{kg} /$ day on day 16 . Serum triglyceride level on day 16 were reduced by compound $\underline{7}, \underline{8}, \underline{9}, \underline{17}$ and $\underline{18}$ by $27 \%$ at $8 \mathrm{mg} / \mathrm{kg} /$ day I.P.

Three compounds $\underline{7}, \underline{9}$ and $\underline{18}$ were selected as being representative compounds to further study in rats their hypolipidemic action [Table 2]. In rats at $8 \mathrm{mg} / \mathrm{kg} /$ cay, compound 9 lowered serum cholesterol levels 328 after 14 days administration orally, whereas compounds $\underline{7}$ and $\underline{18}$ caused at 24-25\% reduction. Nevertheless, these compounds were more potent 
Table 1: In Vivo Hypolipidemic Activity of Carboxy- and Cyanoborane Adducts of Cyclohexylamine and Toluidine in $\mathrm{CF}_{1}$ Male Mice at 8

$\mathrm{mg} / \mathrm{kg} /$ day, I.P. for 16 days.

\begin{tabular}{|c|c|c|c|}
\hline \multirow{2}{*}{$\begin{array}{l}N=6 \\
\text { Compound }\end{array}$} & \multicolumn{3}{|c|}{ Percent of Control ( $\left.X^{-} \pm S . D.\right)$} \\
\hline & $\begin{array}{c}\text { Day } 9 \\
\text { Serum } \\
\text { Cholesterol }\end{array}$ & $\begin{array}{c}\text { Day } 16 \\
\text { Serum } \\
\text { Cholesterol }\end{array}$ & $\begin{array}{c}\text { Day } 16 \\
\text { Serum } \\
\text { Triglyceride }\end{array}$ \\
\hline 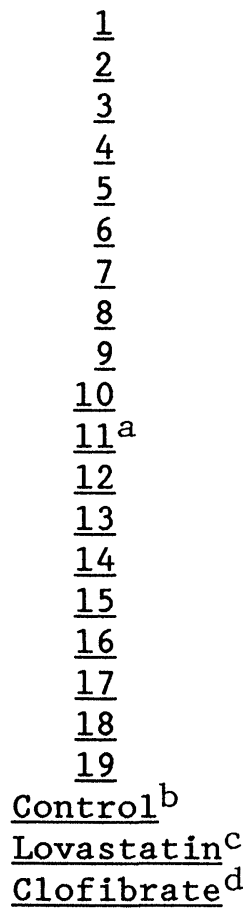 & $\begin{array}{c}85 \pm 4 \\
110 \pm 6 \\
89 \pm 4 \\
90 \pm 2 \\
82 \pm 4 \\
91 \pm 8 \\
87 \pm 2 \\
79 \pm 4 \\
82 \pm 3 \\
90 \pm 9 \\
73 \pm 3^{*} \\
76 \pm 6^{*} \\
83 \pm 3 \\
58 \pm 3^{*} \\
54 \pm 5^{*} \\
73 \pm 2^{*} \\
90 \pm 2 \\
81 \pm 3 \\
87 \pm 3 \\
100 \pm 5^{\mathrm{e}} \\
85 \pm 4 \\
87 \pm 6\end{array}$ & $\begin{array}{c}65 \pm 2^{*} \\
68 \pm 4^{*} \\
79 \pm 1^{*} \\
83 \pm 8 \\
74 \pm 9^{*} \\
84 \pm 2 \\
59 \pm 3^{*} \\
73 \pm 2^{*} \\
62 \pm 5^{*} \\
69 \pm 6^{*} \\
-2 \\
78 \pm 4^{*} \\
68 \pm 6^{*} \\
83 \pm 3^{*} \\
84 \pm 1^{*} \\
67 \pm 4^{*} \\
77 \pm 5^{*} \\
76 \pm 4^{*} \\
87 \pm 3^{*} \\
100 \pm 7^{f} \\
82 \pm 5^{*} \\
78 \pm 6^{*}\end{array}$ & $\begin{array}{l}100 \pm 4 \\
77 \pm 5 \\
84 \pm 11 \\
90 \pm 5 \\
82 \pm 15 \\
78 \pm 9 \\
54 \pm 10^{*} \\
72 \pm 8^{*} \\
66 \pm 7^{*} \\
74 \pm 6^{*} \\
-- \\
84 \pm 5 \\
84 \pm 6 \\
99 \pm 5 \\
112 \pm 9 \\
84 \pm 14 \\
73 \pm 14^{*} \\
61 \pm 15^{*} \\
78 \pm 5^{*} \\
100 \pm 119 \\
86 \pm 7 \\
75 \pm 6^{*}\end{array}$ \\
\hline
\end{tabular}

a Dosed at $4 \mathrm{mg} / \mathrm{kg} /$ day, toxic

b $1 \% \mathrm{CMC}$

c Dosed at $8 \mathrm{mg} / \mathrm{kg} /$ day

$\mathrm{d}$ Dosed at $150 \mathrm{mg} / \mathrm{kg} /$ day

e $125 \mathrm{mg} / \mathrm{dL}$ serum cholesterol

f $128 \mathrm{mg} / \mathrm{dL}$ serum cholesterol

g $137 \mathrm{mg} / \mathrm{dL}$ serum triglyceride

* $p \leq 0.001$, student's " $t$ " test

than lovastatin at $8 \mathrm{mg} / \mathrm{kg} /$ day, gemfibrozil at $90 \mathrm{mg} / \mathrm{kg} /$ day or clofibrate at $150 \mathrm{mg} / \mathrm{kg} /$ day in lowering serum cholesterol levels after 14 days. Compound $\underline{g}$ also most effectively lowered serum triglyceride levels $29 \%$ by day 14 ; yet compounds $\underline{7}$ and 18 only caused $21 \%$ reduction. None of the compounas were as effective as gemfibrozil at $90 \mathrm{mg} / \mathrm{kg} / \mathrm{day}$ with a $38 \%$ reduction of serum triglyceride levels after 14 days. However, the compounds at $8 \mathrm{mg} / \mathrm{kg} /$ day were more effective than lovastatin at $8 \mathrm{mg} / \mathrm{kg} /$ day and approximately equal to clofibrate at 150 $\mathrm{mg} / \mathrm{kg} /$ day. Daily food consumption and total body weight of the treated 
animals were significantly no different than the control group for 14 days.

Table 2: In Vivo Hypolipidemic Activity of Carboxy- and Cyanoborane Adducts of Cyclohexylamine and Toluidine in Sprague-Dawley Male Rats at $8 \mathrm{mg} / \mathrm{kg} /$ day Orally for 14 Days.

\begin{tabular}{|c|c|c|c|c|c|}
\hline Compound & Food $^{a}$ & \multicolumn{2}{|r|}{ Day 7} & \multicolumn{2}{|c|}{ Day 14} \\
\hline \# Consun & mption & Cholesterol & Triglyceride & Cholesterol & Triglyceride \\
\hline 7 & $105 \pm 6$ & $100 \pm 9$ & $72 \pm 7^{*}$ & $75 \pm 10$ & $79 \pm 7$ \\
\hline$\underline{\overline{9}}$ & $102 \pm 7$ & $118 \pm 6$ & $56 \pm 4^{*}$ & $68 \pm 8^{*}$ & $71 \pm 7$ \\
\hline$\underline{18}$ & $100 \pm 7$ & $87 \pm 9$ & $71 \pm 4$ & $76 \pm 7$ & $79 \pm 4$ \\
\hline Controlb & $100 \pm 6$ & $100 \pm 6^{f}$ & $100 \pm 4 \mathrm{~g}$ & $100 \pm 6^{h}$ & $100 \pm 5^{i}$ \\
\hline Lovastatinc & $\ldots$ & $85 \pm 4$ & $91 \pm 5$ & $82 \pm 5$ & $86 \pm 7$ \\
\hline Gemfibrozil & d $91 \pm 5$ & $91 \pm 5$ & $\ldots$ & $82 \pm 7$ & $62 \pm 5$ \\
\hline Clofibrate & $\ldots$ & $89 \pm 7$ & $83 \pm 6$ & $86 \pm 5$ & $74 \pm 7$ \\
\hline
\end{tabular}

a Control $=22.1 \pm 1.3 \mathrm{~g} /$ day/rat

b $18 \mathrm{CMC}$

c Dosed at $8 \mathrm{mg} / \mathrm{kg} /$ day

$\mathrm{d}$ Dosed at $90 \mathrm{mg} / \mathrm{kg} /$ day

e Dosed at $150 \mathrm{mg} / \mathrm{kg} /$ day

f $73 \mathrm{mg} / \mathrm{dL}$ total serum cholesterol

g $75 \mathrm{mg} / \mathrm{dL}$ total serum cholesterol

h $111 \mathrm{mg} / \mathrm{dL}$ serum triglyceride

i $112 \mathrm{mg} / \mathrm{dL}$ serum triglyceride

* $p \leq 0.001$, Student's " $t$ " test

Further tissue lipid extraction studies showed that in rats treated 14 days with compounds $\underline{7}, \underline{9}$ or $\underline{18}$ [Table 3], liver, small intestinal mucosa and aorta lipid content was reduced. The triglyceride content was reduced more consistently than the cholesterol content in all three tissues. In the aorta tissue neutral lipids and phospholipid content was also reduced after 14 days treatment. Protein and cholesterol content of the aorta tissue was reduced after treatment with compound 9 . Total lipid excretion into the feces was not affected by treatment with the compounds. Fecal triglycerides were elevated significantly by all three compounds; however, phospholipids excretion was reduced [Table 3]. Examination of the lipid content of the rat lipoproteins after 14 days administration showed that all three compounds lowered cholesterol content in the chylomicron and the LDL fraction. The HDL cholesterol content was significantly elevated 2 to 3 fold [Table 4] by all three compounds. Triglyceride content was elevated in the chylomicron fraction after treatment with compounds $\underline{9}$ and $\underline{18}$. Triglyceride content was elevated in the VLDL fraction after treatment with compound $\underline{7}$. HDL triglyceride content was elevated by treatment with compounds $\underline{I}$ and $\underline{9}$. Neutral lipid content of the lipoprotein fractions was not affected by any of the compounds. Phospholipid content of VLDL fraction was reduced by all three compourds while phospholipid content of LDl fraction was 
Table 3 In Vivo Effects of Carboxy- and Cyanoborane Adducts of Cyclohexylamine and Toluidine on Tissue Lipids in Sprague-Dawley Male Rats After 14 Days at $8 \mathrm{mg} / \mathrm{kg} /$ day, Orally $N=8$ Percent of Control (X \pm S.D.)

\begin{tabular}{|c|c|c|c|c|c|c|}
\hline $\begin{array}{l}\mathrm{Mg} \text { of } \\
\text { Extra }\end{array}$ & $\begin{array}{l}\text { Lipid } \\
\text { acted }\end{array}$ & holesterol & Triglycerides & $\begin{array}{l}\text { Neutral } \\
\text { Lipids }\end{array}$ & $\begin{array}{l}\text { Phospho- } \\
\text { lipids }\end{array}$ & Protein \\
\hline \multicolumn{7}{|c|}{ Liver } \\
\hline Control & $100 \pm 4^{a}$ & $100 \pm 5^{b}$ & $100 \pm 7^{c}$ & $100 \pm 6^{d}$ & $100 \pm 7^{e}$ & $100 \pm 6^{f}$ \\
\hline$\underline{7}$ & $86 \pm 6$ & $93 \pm 5$ & $64 \pm 5^{*}$ & $101 \pm 6$ & $104 \pm 8$ & $81 \pm 5$ \\
\hline$\underline{9}$ & $86 \pm 2$ & $102 \pm 8$ & $88 \pm 6$ & $97 \pm 6$ & $104 \pm 5$ & $104 \pm 6$ \\
\hline$\underline{18}$ & $96 \pm 3$ & $109 \pm 6$ & $164 \pm 7^{*}$ & $98 \pm 5$ & $91 \pm 8$ & $110 \pm 8$ \\
\hline \multicolumn{7}{|c|}{ Sma11 Intestine } \\
\hline Control & $100 \pm 8 g$ & $100 \pm 7^{h}$ & $100 \pm 6^{i}$ & $100 \pm 6^{j}$ & $100 \pm 6^{k}$ & $100 \pm 7^{1}$ \\
\hline 1 & $76 \pm 11^{*}$ & $94 \pm 6$ & $55 \pm 4^{*}$ & $105 \pm 8$ & $93 \pm 6$ & $117 \pm 5$ \\
\hline$\overline{9}$ & $93 \pm 16$ & $117 \pm 7$ & $67 \pm 5^{*}$ & $98 \pm 6$ & $79 \pm 4^{*}$ & $115 \pm 5$ \\
\hline$\underline{18}$ & $88 \pm 7$ & $112 \pm 8$ & $87 \pm 6$ & $91 \pm 5$ & $66 \pm 5^{*}$ & $119 \pm 5$ \\
\hline \multicolumn{7}{|c|}{ Aorta } \\
\hline Control & $100 \pm 4^{m}$ & $1 C 0 \pm 5^{n}$ & $100 \pm 6^{\circ}$ & $100 \pm 5 p$ & $100 \pm 6 q$ & $100 \pm 6^{r}$ \\
\hline$\underline{7}$ & $88 \pm 12$ & $112 \pm 6$ & $86 \pm 4$ & $58 \pm 4^{*}$ & $91 \pm 6$ & $94 \pm 6$ \\
\hline$\underline{\overline{9}}$ & $66 \pm 2^{*}$ & $79 \pm 4 *$ & $81 \pm 4^{*}$ & $49 \pm 5^{*}$ & $82 \pm 7$ & $65 \pm 6^{*}$ \\
\hline$\underline{18}$ & $101 \pm 12$ & $101 \pm 5$ & $99 \pm 6$ & $89 \pm 5$ & $54 \pm 5^{*}$ & $89 \pm 5$ \\
\hline \multicolumn{7}{|c|}{ Feces } \\
\hline Control & $100 \pm 8^{s}$ & $100 \pm 6^{t}$ & $100 \pm 6^{\mathrm{u}}$ & $100 \pm 8^{v}$ & $100 \pm 8^{W}$ & $100 \pm 6^{x}$ \\
\hline 1 & $99 \pm 3$ & $87 \pm 7$ & $148 \pm 9^{*}$ & $99 \pm 7$ & $57 \pm 5^{*}$ & $104 \pm 6$ \\
\hline$\underline{9}$ & $101 \pm 10$ & $c 5 \pm 9$ & $155 \pm 8^{*}$ & $97 \pm 8$ & $65 \pm 5^{*}$ & $115 \pm 6$ \\
\hline$\underline{18}$ & $104 \pm 9$ & $87+8$ & $173 \pm 8^{*}$ & $100 \pm 7$ & $45 \pm 4^{*}$ & $110 \pm 5$ \\
\hline
\end{tabular}

per gram wet tissue

a $50.5 \mathrm{mg}$ lipid

b $9.18 \mathrm{mg}$ cholesterol

c $6.37 \mathrm{mg}$ triglyceride

d $15.70 \mathrm{mg}$ neutral lipid

e $27.19 \mathrm{mg}$ phospholipid

f $12.02 \mathrm{mg}$ protein

g $68.20 \mathrm{mg}$ lipid

h $12.02 \mathrm{mg}$ cholesterol

i $11.20 \mathrm{mg}$ triglyceride

j $16.98 \mathrm{mg}$ neutral lipid

k $20.06 \mathrm{mg}$ phospholipid

$142.0 \mathrm{mg}$ protein

* $p \leq 0.001$, Student's " $t$ " test
In $67.5 \mathrm{mg}$ lipid

n $5.77 \mathrm{mg}$ cholesterol

- $9.85 \mathrm{mg}$ triglyceride

p $15.28 \mathrm{mg}$ neutral lipid

q $28.8 \mathrm{mg}$ phospholipid

r $11.71 \mathrm{mg}$ protein

s $11.58 \mathrm{mg}$ lipid

$t \quad 2.84 \mathrm{mg}$ cholesterol

$\mathrm{u} 1.86 \mathrm{mg}$ triglyceride

$\mathrm{v} 3.39 \mathrm{mg}$ neutral lipid

w $5.70 \mathrm{mg}$ phospholipid

x $6.99 \mathrm{mg}$ protein

elevated approximately two fold. The protein content of the chylomicron fraction was reducea after 14 day treatment.

The mode of action of the derivatives on rate limiting enzymes involved in liver lipid metabolism showed that the compounds were not HMG-CoA reductase inhibitors. Rather these compounds reduced the activites of cytoplasmic acetyl CoA synthetase and ATP-dependent citrate lyase. Compound $\underline{Z}$ and $\underline{9}$ caused a moderate reduction of $11-18 \%$ in acyl CoA cholesterol acyl transferase activity. Neutral cholesterol ester 
Table 4: In Vivo Effects of Carboxy- and Cyanoborane Adducts of Cyclohexylamine and Toluidine on the Lipid Content of Serum Lipoproteins in Sprague Dawley Male Rats After 14 Days at $8 \mathrm{mg} / \mathrm{kg} /$ day, Orally $\mathrm{N}=4$ Percent of Control (X + S.D.) Cholesterol Triglycerides Neutral Phospho- Protein

\begin{tabular}{|c|c|c|c|c|c|}
\hline \multicolumn{6}{|c|}{ Chylomicron } \\
\hline Control & $100 \pm 6^{a}$ & $100 \pm 7^{b}$ & $100 \pm 7^{c}$ & $100 \pm 6^{d}$ & $100 \pm 5^{e}$ \\
\hline 1 & $67 \pm 5^{*}$ & $105 \pm 7$ & $92 \pm 5$ & $86 \pm 5$ & $79 \pm 5$ \\
\hline$\underline{9}$ & $67 \pm 4^{*}$ & $158 \pm 8^{*}$ & $96 \pm 6$ & $85 \pm 6$ & $81 \pm 6$ \\
\hline $1 \overline{8}$ & $50 \pm 6^{*}$ & $136 \pm 9^{*}$ & $91 \pm 6$ & $104 \pm 6$ & $80+7$ \\
\hline \multicolumn{6}{|l|}{ VLDL } \\
\hline Control & $100 \pm 4^{f}$ & $100 \pm 5 g$ & $100 \pm 7^{h}$ & $100 \pm 7^{i}$ & $100 \pm 5^{j}$ \\
\hline$\underline{7}$ & $104 \pm 5$ & $123 \pm 6$ & $105 \pm 6$ & $62 \pm 5^{*}$ & $107 \pm 6$ \\
\hline$\underline{9}$ & $118 \pm 5$ & $99 \pm 5$ & $107 \pm 6$ & $44 \pm 4^{*}$ & $76 \pm 6^{*}$ \\
\hline 18 & $92 \pm 4$ & $91 \pm 8$ & $107 \pm 5$ & $43 \pm 4^{*}$ & $93 \pm 5$ \\
\hline \multicolumn{6}{|l|}{ LDL } \\
\hline Control & $100 \pm 5^{k}$ & $100 \pm 6^{1}$ & $100 \pm 6^{\mathrm{m}}$ & $100 \pm 6^{n}$ & $100 \pm 8^{\circ}$ \\
\hline$\underline{7}$ & $65 \pm 5^{4}$ & $114 \pm 6$ & $98 \pm 7$ & $202 \pm 7^{*}$ & $89 \pm 6$ \\
\hline 9 & $50 \pm 4^{*}$ & $101 \pm 7$ & $100 \pm 7$ & $182 \pm 8^{*}$ & $133 \pm 7^{*}$ \\
\hline 18 & $63 \pm 4^{*}$ & $110 \pm 6$ & $99 \pm 6$ & $214 \pm 8^{*}$ & $96 \pm 8$ \\
\hline \multicolumn{6}{|l|}{ HDL } \\
\hline Control & $100 \pm 6 \mathrm{P}$ & $100 \pm 7 q$ & $100 \pm 6^{I}$ & $100 \pm 7^{s}$ & $100 \pm 6^{t}$ \\
\hline I & $348 \pm 10^{*}$ & $127 \pm 5^{*}$ & $93 \pm 4$ & $110 \pm 7$ & $166 \pm 6^{*}$ \\
\hline$\underline{9}$ & $371 \pm 9^{*}$ & $116 \pm 6$ & $89 \pm 5$ & $107 \pm 6$ & $166 \pm 6^{*}$ \\
\hline 18 & $235 \pm 9^{*}$ & $129 \pm 6^{*}$ & $92 \pm 6$ & $81 \pm 7$ & $167 \pm 5^{*}$ \\
\hline
\end{tabular}

\begin{tabular}{|c|c|}
\hline $337 \mu \mathrm{g}$ cholesterol $/ \mathrm{mL}$ serum & k $210 \mu \mathrm{g}$ cholesterol/mL serum \\
\hline $420 \mu \mathrm{g}$ triglyceride/mL serum & $145 \mu \mathrm{g}$ triglyceride/mL serum \\
\hline $67 \mu \mathrm{g}$ neutral lipid/mL serum & $\mathrm{m} 10 \mu \mathrm{g}$ neutral lipid/mL serum \\
\hline $149 \mu \mathrm{g}$ phospholipid/mL serum & $41 \mu \mathrm{g}$ phospholipid/mL serum \\
\hline $184 \mu \mathrm{g}$ protein $/ \mathrm{mL}$ serum & $122 \mu \mathrm{g}$ protein $/ \mathrm{mL}$ serum \\
\hline $190 \mu \mathrm{g}$ cholestercl $/ \mathrm{mL}$ serum & $544 \mu \mathrm{g}$ cholesterol $/ \mathrm{mL}$ serum \\
\hline $22 \mu \mathrm{g}$ triglycerice/mL serum & $630 \mu \mathrm{g}$ triglyceride/mL serum \\
\hline $98 \mu \mathrm{g}$ neutral lifid/mL serum & $27 \mu \mathrm{g}$ neutral lipid/mL serum \\
\hline $26 \mu \mathrm{g}$ phospholipid/mL serum & $153 \mu \mathrm{g}$ phospholipid/mL serum \\
\hline $\begin{array}{l}\mathrm{j} \quad 50 \mu \mathrm{g} \text { protein/mL serum } \\
{ }^{*} p \leq 0.001 \text {, Student's " } t \text { " test }\end{array}$ & t $657 \mu g$ protein $/ \mathrm{mL}$ serum \\
\hline
\end{tabular}

hydrolase activity was reduced by compound $I$ by 448 , but the other two compounds only afforded $12-15 \%$ reduction. Cholesterol-7 $\alpha$-hydroxylase activity was significantly elevated by all three compounds. Acyl CoA carboxylase activity was suppressed $17 \%$ by compound $\underline{Z}$ and $29 \%$ by compounds $\underline{9}$ and 18 . sn-Glycerol-3-phopshate acyl transferase activity was reduced $15 \%$ by compound $\underline{9}$ and $19 \%$ by compound 18 . Phosphatidyate phosphohydrolase activity was reduced markedly $76 \%$ by compound $\underline{7}, 82 \%$ by compound $\underline{9}$ and $88 \%$ by compound 18 . Hepatic lipoprotein lipase activity was reduced $29 \%$ by compound $I$ and $19 \%$ by compound $\underline{18}$. 
Table 5: In Vitro Inhibtion of Liver Lipid Enzyme Activies by Carboxyand Cyanoborane Adducts of Cyclohexylamine and Toluidine

$\bar{N}=6$

Percent of Control $(X \pm S . D$.

Enzyme Assay

Compound 7

Acetyl CoA Synthetase

Control $25 \mu \mathrm{M} \quad 50 \mu \mathrm{M} \quad 100 \mu \mathrm{M}$

ATP-dependent Citrate Lyase

HMG-CoA Reductase

Acyl CoA Cholesterol

Acyl Transferase

Neutral Cholesterol Ester

Hydrolase

$\begin{array}{llll}100 \pm 7^{\mathrm{a}} & 98 \pm 7 & 88 \pm 6 & 71 \pm 6^{*}\end{array}$

$100 \pm 5^{\mathrm{b}} \quad 92 \pm 4 \quad 73 \pm 5^{*} \quad 67 \pm 4^{*}$

$100 \pm 7^{\mathrm{C}} \quad 107 \pm 6 \quad 108 \pm 7 \quad 129 \pm 7^{*}$

Cholesterol-7-alpha-hydroxylase

Acyl CoA Carboxylase

$100 \pm 6^{\mathrm{d}} \quad 122 \pm 6 \quad 84 \pm 6 \quad 82 \pm 7$

sn-Glycerol-3-Phosphate

Acyl Transferase

$100 \pm 5^{\mathrm{e}} \quad 71 \pm 6 \quad 66 \pm 6^{*} \quad 56 \pm 5^{*}$

$100 \pm 6$ f $122 \pm 5 * 125 \pm 6^{*} 150 \pm 6$ *

$100 \pm 58 \quad 92 \pm 5 \quad 84 \pm 5 \quad 83 \pm 6$

Phosphatidylate

Phosphohydrolase

$100 \pm 6^{\text {h }} \quad 115 \pm 6 \quad 109 \pm 6 \quad 101 \pm 5$

Lipoprotein Lipase

$100 \pm 5^{i} \quad 108 \pm 6 \quad 90 \pm 6 \quad 24 \pm 5^{*}$

$100 \pm 6 j \quad 117 \pm 6 \quad 102 \pm 4 \quad 71 \pm 5^{*}$

$\mathrm{N}=6$

Compound: $\underline{9}$

Control $25 \mu \mathrm{M} \quad 50 \mu \mathrm{M} \quad 100 \mu \mathrm{M}$

Acetyl CoA Synthetase

$\begin{array}{lccc}100 \pm 7^{\mathrm{a}} & 99 \pm 6 & 84 \pm 5 & 80 \pm 5^{*} \\ 100 \pm 5^{\mathrm{b}} & 115 \pm 6 & 114 \pm 7 & 78 \pm 6^{*} \\ 100 \pm 7^{\mathrm{c}} & 135 \pm 7^{*} & 132 \pm 6^{*} & 135 \pm 4^{*} \\ 100 \pm 6^{\mathrm{d}} & 118 \pm 5 & 93 \pm 4 & 89 \pm 5 \\ 100 \pm 5^{\mathrm{e}} & 94 \pm 6 & 89 \pm 4 & 88 \pm 6 \\ 100 \pm 6^{\mathrm{f}} & 205 \pm 8^{*} & 237 \pm 8^{*} & 198 \pm 7^{*} \\ 100 \pm 5 \mathrm{~g} & 79 \pm 5^{*} & 75 \pm 4^{*} & 71 \pm 5^{*} \\ 100 \pm 6^{\mathrm{h}} & 107 \pm 6 & 95 \pm 4^{*} & 85 \pm 4 \\ 100 \pm 5^{\mathrm{i}} & 46 \pm 5^{*} & 31 \pm 5^{*} & 18 \pm 3^{*} \\ 100 \pm 6 \mathrm{j} & 125 \pm 5 & 114 \pm 4 & 96 \pm 4\end{array}$

ATP-dependent Citrate Lyase

HMG CoA Reductase

Acyl CoA Cholesterol

Acyl Transferase

Neutral Cholesterol Ester

Hydrolase

Cholesterol-7-alpha-hydroxylase

Acyl CoA Carboxylase

sn-Glycerol-3-Phosphate

Acyl Transferase

Phosphatidylate

Phosphohydrolase

Lipoprotein Lipase 
B.S. Burnham, S.Y. Chen, A. Sood,

B.F. Spielvogel and I.H. Hall

Table 5 (Continued):

$\mathrm{N}=6$

Enzyme Asdsay

Compound: $\underline{18}$

Acetyl CoA synthetase

ATP-dependent Citrate Lyase

HMG CoA Reductase

Acyl CoA Cholesterol

Acyl Transferase

Neutral Cholesterol Ester

Hydrolase

Control $25 \mu \mathrm{M} \quad 50 \mu \mathrm{M} \quad 100 \mu \mathrm{M}$

Cholesterol-7-alpha-hydroxylase

Acyl CoA Carboxylase

sn-Glycerol-3-Phosphate

Acyl Transferase

Phosphatidylate

Phosphohydrolase

Lipoprotein Lipase

$\begin{array}{lccc}100 \pm 7^{\mathrm{a}} & 104 \pm 7 & 96 \pm 5 & 66 \pm 5^{*} \\ 100 \pm 5^{\mathrm{b}} & 125 \pm 6 & 123 \pm 4 & 101 \pm 5 \\ 100 \pm 7^{\mathrm{c}} & 116 \pm 5 & 113 \pm 8 & 96 \pm 5 \\ 100 \pm 6^{\mathrm{d}} & 108 \pm 7 & 103 \pm 4 & 97 \pm 6 \\ 100 \pm 5^{\mathrm{e}} & 109 \pm 5 & 106 \pm 4 & 85 \pm 6 \\ 100 \pm 6^{\mathrm{f}} & 179 \pm 6^{*} & 241 \pm 5^{*} & 250 \pm 6^{*} \\ 100 \pm 5 \mathrm{~g} & 84 \pm 3 & 82 \pm 6 & 71 \pm 4^{*} \\ 100 \pm 6^{\mathrm{h}} & 98 \pm 6 & 89 \pm 5 & 81 \pm 4^{*} \\ 100 \pm 5^{\mathrm{i}} & 24 \pm 3^{*} & 17 \pm 2^{*} & 12 \pm 2^{*} \\ 100 \pm 6 \mathrm{j} & 110 \pm 6 & 102 \pm 5 & 81 \pm 4^{*}\end{array}$

a $28.5 \mathrm{mg}$ acetyl CoA formed/g wet tissue b $30.5 \mathrm{mg}$ citrate hydrolyzed/g wet tissue c $384900 \mathrm{dpm}$ cholesterol formed/g wet tissue d 224000 $\mathrm{dpm} / \mathrm{mg}$ of microsumal protein e $56436 \mathrm{dpm} / \mathrm{mg}$ wet tissue $\mathrm{f} 4808 \mathrm{dpm} / \mathrm{mg}$ of microsomal protein g $537800 \mathrm{dpm} / \mathrm{mg}$ wet tissue h $302010 \mathrm{dpm} / \mathrm{mg}$ wet tissue $i 16.7 \mu g P_{i}$ released/g wet tissue $j 278538 \mathrm{dpm} / g$ wet tissue * $p \leq 0.001$, Student's " $t$ " test

DISCUSSION

These studies have shown that carboxy- and cyanoborane adducts of cyclohexylamine and toluidine possessed good hypolipidemic acitivity in rodents. They were approximately equal in their ability to lower serum cholesterol and triglyceride levels in mice at $8 \mathrm{mg} / \mathrm{kg} /$ day as the heterocyclic amine boranes. Thus, it appears the nitrogen does not have to be in the aromatic ring to retain good hypolipidemic activity. Like the heterocyclic amine boranes there was no significant difference between carboxyboranes and cyanoboranes as far as their ability to lower serum cholesterol and triglycerides after 16 days administration I.P. in mice. However, it should be noted that compound $\underline{Z}$ did not afford as good activity in rats as it did in mice, although its magnitude of serum lipid reduction was still comparable to the standards lovastatin and clofibrate. This type of species variation has been noted before with boron derivatives[7]. Like many of the boron derivative these new compounds lowered cholesterol, triglyceride and phospholipid content in the major organs. What is most important is the fact that compound $\underline{9}$ was very effective in lowering cholesterol content within the rat aorta wall after 14 days administration. These adducts were very impressive with regard to their effects on lipoprotein lipid content. An ideal hypolipidemic agent should lower cholesterol content of the VLDL and LDL fraction since these lipoproteins are responsible for conducting cholesterol to the peripheral tissue including the arterial walls, whereas HDL cholesterol content should be high so that cholesterol may be conducted back to the liver for biliary excretion. These derivatives were able to successfully lower VLDL and LDL cholesterol content and elevate HDL cholesterol content. In fact the elevation HDL cholesterol 
content afforded by these agents is much higher than the standards lovastatin with a 298 increase [34] or gemfibrozil with a $104 \%$ increase in rat HDL-cholesterol content[11]. Supposedly, a clinical agent which lowers LDL- and VLDL-cholesterol content but elevates HDL-cholesterol content protects man from mycocardial infarction[35]. The mode of action of the derivatives on regulatory enzyme activities involved in lipid metabolism appeared to be at site which regulated cytoplasmic formation of acetyl-CoA for cholesterol and fatty acid synthesis. These agents were not HMG-CoA reductase inhibitors whereas the heterocyclic amine-carboxy- and cyanoboranes were potent inhibitors. The amine-borane adducts of cyclohexylamine and toluidine accelerated cholesterol-7 $\alpha$-hydroxylase activity more than the heterocyclic amine boranes. This would suggest that the former compounds probably accelerate the conversion of cholesterol to bile acids theoretically increasing cholesterol clearance from the body. These derivative marginally inhibited acyl-cholesterol acyl transferase activity which should reduce cholesterol ester storage in tissues. In aorta walls this enzyme plays a role in depositing cholesterol esters in foam cells and increased plaque growth; thus inhibition of its activity should slow the process. It was hoped that the agents would accelerate cholesterol ester hydrolase activity. This enzyme breaks cholesterol esters down to free cholesterol which can be picked up by HDL to be conducted back to the liver via the reverse cholesterol transport mechanism for biliary excetion of cholesterol. This enzyme level is low in man but it is inducable by selective hypolipidemic agents to cause more clearnce of aorta wall lipids[36]. The heterocyclic amine-boranes were able to increase the activity of cholesterol ester hydrolase. The neutral lipid and triglyceride pathways were inhibited by these derivatives at the site of phophatidylate phosphohydrolase, so that triglyceride can not be formed from phospholipids. The other regular enzyme of the triglyceride pathway, sn-glycerol-3-phosphate acyl transferase, was marginally inhibited by compounds $\underline{9}$ and $\underline{18}$. These adducts were more potent in this respect than the heterocyclic amineboranes. Since hepatic lipoprotein lipase activity was also inhibited by the amine borane adducts of cyclohexylamine and toluidine, the inhibition of these three enzyme activities should be of a magnitude to lower triglyceride levels in the blood and tissue. Furthermore, some accelerated loss of triglycerides occurred in the feces, although bile excretion of triglycerides is considered to be of a small magnitude under normal conditions.

In conclusion, the amine-borane adducts of cyclohexylamine and toluidine maintain good hypolipidemic activity and achieve alteration in lipid metabolism and clearance which are desirable. Further investigation is warrented to evaluate such derivatives for clinical trails.

\section{REFERENCES}

1. Hall, I.H., Spielvogel, B.F., Das, M.K., and McPhail, A.T. (1981) J. Pharm. Sci. 7C, 339.

2. Hall, I.H., Das, M.K., Harchelroad, Jr., F., Wisian-Neilson, P., McPhail, A.T. and Spielvogel, B.F., (1981) J. Pharm. Sci. 70, 339.

3. Sood, A., Sood, C.K., Spielvogel, B.F., Hall, I.H., and Wong, O.T., (1991) Arch Pharm. 324, 423. 
4. Hall, I.H., Spielvogel, B.F., Sood, A., Ahmed, F., and Jafri, S. (1987) J. Pharm. Sci. 76, 359.

5. Hall, I.H., Chen, S.Y., Rajendran, K.G., Sood, A., Spielvogel, B.F., and Shih, J. (1994) Environ. Health Perspect 102, 21.

6. Sood, A., Sood, C.K., Spielvogel, B.F., and Hall, I.H. (1990) European. $\therefore$. Med. Chem. 25, 301.

7. Hall, I.H., Sood, A., and Spielvogel, B.F., (1991) Biomed. Pharmacother 45,333 .

8. Hall, I.H., Brotherton, R.J.,Griffin, T.S., Sood, A., and Spielvogel, B.F. (1991.) Biomed. Biochim. Acta 50, 1007.

9. Sood, A., Sood, C.K., Spielvogel, B.F., Hall, I.H., and Wong, O.T (1992) J. Pharm. Sci. 81, 458.

10. Hall. I.H., Wong, O.T., Sood, A. Spielvogel, B.F., and Morse, K.W. (1992) Pharmacol. Res. Commun. 25, 259.

11. Hall, I.H., Burnham, B.S., Shaw, B.R., Rajendran, K.G., Chen, S.Y., Sood, A., and Spielvogel, B.F. (1993) Biomed. Pharmacotherapy 47, 79 .

12. Hall, I.H., Reynolds, D.J., Chang, J., Spielvoge1, B.F., Griffin, T.S. and Docks, E.L. (1991) Arch. Pharm. 324, 573.

13. Burnham, B.S., Chen, S.Y., Sood, A., Spielvogel, B.F., and Hall, I.H. (1995) die Pharmazie, submitted.

14.. Ness, A.T., Pastewka, J.V., and Peacock, A.C. (1959) Clin. Chem. Acta. 10, 229.

15. Hall, I.H., Cocolas, G.H., and Voorstad, P.J. (1984) J. Pharm. Sci. 73,812

16. Bligh, E.G., anci Dyer, W.J. (1959) J. Biochem. Physiol. 37, 911.

17. Folch, J., Lees, M., and Stanley, G.H.C. (1957) J. Biol. Chem. 226, 497.

18. Bragdon, J.H. (1951) J. Biol. Chem. 190, 513.

19. Stewart, C.P., and Hendry, E.G. (1935) J. Biochem. 29, 1688.

20. Lowry, O.H., Rosebrough, N.J., Farr, A.L., and Randa11, R.J. (1951) J. Biol. Chem. 193, 263.

21. Have1, R.L., Eder, H.A., and Bragdon, J.M. (1955) J. Clin. Invest. 34,1345 .

22. Mookerjea, E.S., Parks, C.E., and Kuksis, A. (1975) Lipids 10, 374.

23. Hoffmman, M., Weiss, L., and Wieland, O.H. (1978) Anal. Biochem. 84, 441.

24. Goodridge, A.G.(1973) J. Biol. Chem. 248, 4318.

25. Haven, G.T., Krzemian, J.R., and Nguyen, T.T. (1973) Res. Commun. Chem. Path. Pharmacol. 6, 253.

26. Wada, F., Hirata, K., and Sakameto, Y. (1989) J. Biochem. 65, 171.

27. Shefer, S., Hauser, S., and Mosbach, E.H. (1978) J. Lipid Res.9, 328 .

28. Balasubramaniam, S., Mitropoulos, K.A., and Venkatesam, S. (1978) European J. Biochem. 90, 377.

29. Hal1, I.H., Wong, O.T., and Wyrick, S.D. (1988) Pharm. Res. 5, 413.

30. Greenspan, M.D., and Lowenstein, J.M. (1968) J. Biol. Chem. 243, 6273.

31. Lamb, R.G., Wyrick, S.D., and Piantadosi, C. (1977) Athersclerosis $27,147$.

32. Mavis, R.D., Jacob, N., Finkelstein, J.N., and Hall, B.P. (1978) J. Lipid Res. 19, 467.

33. Chait, A., Iverius, P.H., and Brunzell, J.D. (1982) J. Clin. Invest. 69,490 . 
34. Hall, I.H., Wong, O T., Reynolds, D.J., and Simlot, R. (1993) J. Pharm. Sci. 82, 565.

35. Miettinen, T.A., Huttunen, J.K., Strandberg, T., Naukkarinen, V., Mattila, S., and Kumlin T. (1981) Lancet 2, 478.

36. Wong, O.T., Williams, Jr. W.L., Oswald, B.S., and Hall. I.H. (1992)

Res. Commun. Chem. Path. and Pharm. 76, 3.

Received: April 7, 1995 - Accepted: May 5, 1995 - Received camera-ready revised version: May 23, 1995 